\title{
Right circumcaval ureter and double right renal vein in the Brazilian shorthair cat (Felis catus): two case reports
}

\author{
M. Abidu-Figueiredo ${ }^{1}$, A.V. Stocco' ${ }^{1}$, C.A. Santos-Sousa ${ }^{1,2}$, P. Souza Junior ${ }^{3}$, \\ L.A.S. Pires ${ }^{4}$, M.A. Babinski ${ }^{4}$ \\ 'Universidade Federal Rural do Rio de Janeiro, Brazil \\ 2Universidade Federal do Acre, Brazil \\ 3Universidade Federal do Pampa, Brazil \\ ${ }^{4}$ Universidade Federal Fluminense, Brazil, Brazil
}

[Received: 29 June 2018; Accepted: 19 August 2018]

\begin{abstract}
Variations of the renal veins are well described in the literature, although variations concerning the ureter are considered a rare finding in cats. The circumcaval ureter is one of the rarest variations of the ureter and is characterised by a loop of the ureter posterior to the caudal vena cava. This variant is also known as preureteral vena cava and retrocaval ureter. It is thought to be caused by a deviation during embryonic development of the aforementioned vein. Due to its rarity, there are scarce reports of the circumcaval ureter in cats, and its association with two renal veins makes it less common as well. These variations should be preoperatively identified in order to avoid complications in kidney transplants, ureteral surgeries and cystoscopies, for instance. The present work aims to report two cases of a circumcaval ureter with two renal veins in two different Brazilian shorthair cats (Felis catus). (Folia Morphol 2019; 78, 2: 433-436)
\end{abstract}

Key words: anatomical variations, circumcaval ureter, preureteral vena cava, retrocaval ureter, cat, renal vein

\section{INTRODUCTION}

The renal pedicle is composed by the ureter, one renal vein and one renal artery. The ureters are retroperitoneal conduits that originate from the renal pelvis. The right and left ureters run a lateral, caudal and ventral course in order to reach the urinary bladder $[2,9]$.

In domestic mammalian species, both the right and left renal veins drain their contents into the caudal vena cava. However, the number, course, and site of drainage of the renal veins are largely variable. Anatomical variations of the renal veins are occasionally found in radiological studies and the dissecting room, as knowledge of such variants is necessary prior to the execution of vascular procedures involving the renal pedicle and is essential to avoid confusion during imaging exams $[4,8]$.

On the other hand ureteral variations, such as the circumcaval ureter (also known as the retrocaval ureter) are rarely reported in mammalian species other than humans. This variant has been associated with urinary tract infections, haematuria and hydronephrosis in humans. However, there is debate whether this variation can lead to symptoms in animals such as cats, due to its rarity. Understanding the congenital abnormalities of the ureter may prevent iatrogenic errors or confusion during surgery and exams such as cystoscopy [2-5].

The work presented herein aims to report the presence of doubled right renal veins associated with 


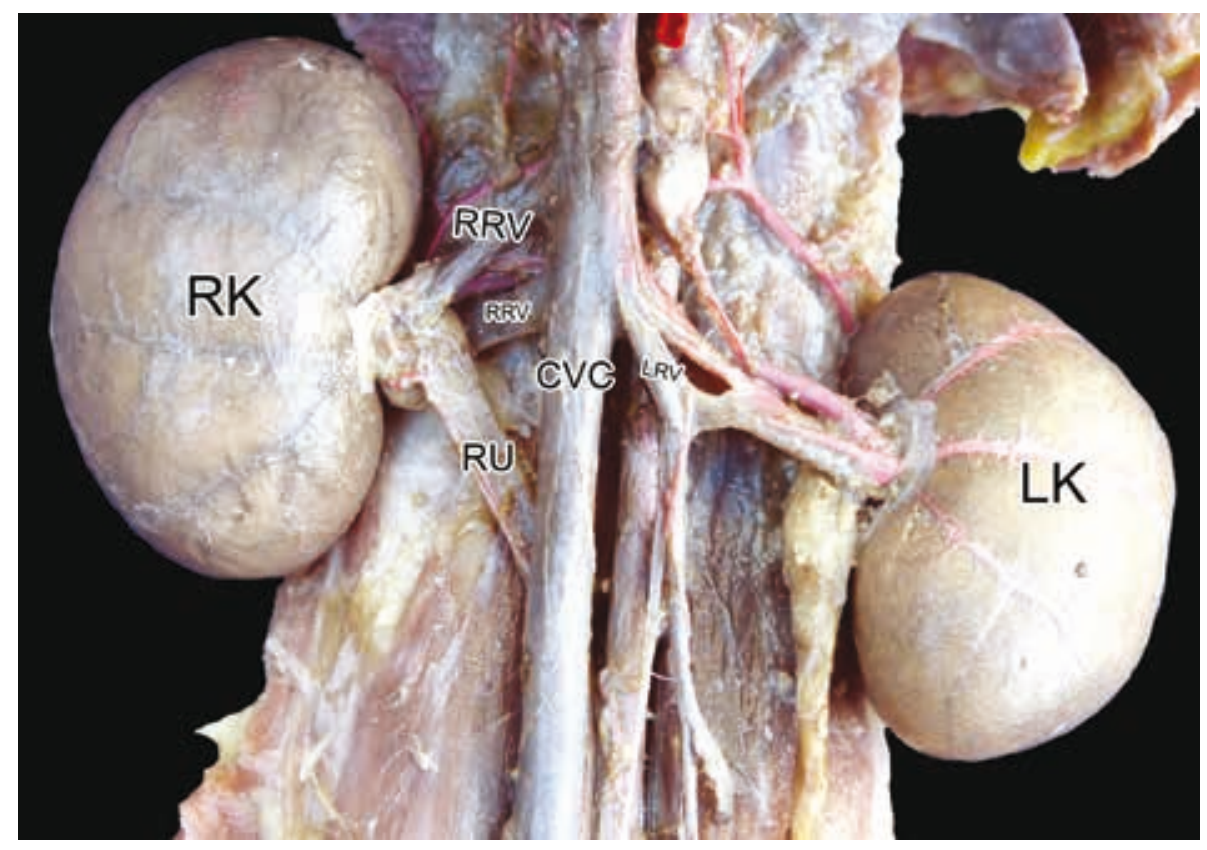

Figure 1. Ventral view of the first cat. The right circumcaval ureter (RU) can be seen together with the right renal veins (RRV); RK — right kidney; LK — left kidney; CVC — caudal vena cava; LRV — left renal vein.

a circumcaval ureter in two Brazilian shorthair cats and discuss its clinical significance.

\section{CASE REPORT}

The current research was approved by the Research Ethics Committee of Rio de Janeiro Federal Rural University (23083.005334/2009-49).

Two male Brazilian shorthair cats obtained from the Rio de Janeiro Federal Rural University Veterinary Hospital necropsy unit were previously identified and positioned in the right lateral recumbency. An incision was performed on the thorax and their $6^{\text {th }}$ and $7^{\text {th }}$ ribs were removed. Afterwards, their thoracic aorta was minimally dissected, cannulated and further injected with saline solution, followed by a $10 \%$ formaldehyde solution.

Their vascular system was then filled with coloured stained Petrolatex S-65 solution (Refinaria Duque de Caxias-REDUC-Petrobras, Duque de Caxias-RJ) mixed with red dye (Suvinilxadrez ${ }^{\circledR}$ ).

Subsequently, the cadavers were immersed in a low-density polyethylene cardboard box with a 500 -litre capacity containing a $10 \%$ formaldehyde solution to finalise the fixation and latex polymerisation process. Seven days after the latex injection, the cadavers were washed in running water and the abdominal cavity was dissected for teaching purposes. Measurements of the kidney and its vessels were performed with the aid of a digital calliper.
After dissection of the retroperitoneal organs, it was observed on the first cat two right renal veins and a right type 1 circumcaval ureter (Fig. 1). The length of both veins were measured with the aid of a digital calliper, and the most cranial and ventral vein measured $1.45 \mathrm{~cm}$, while the most caudal and dorsal vein measured $1.57 \mathrm{~cm}$. The right kidney was $3.61 \mathrm{~cm}$ long, $2.32 \mathrm{~cm}$ wide and $1.97 \mathrm{~cm}$ thick.

On the second cat, two right renal veins were also observed in association with a right type 1 circumcaval ureter (Fig. 2). The length of the most cranial and ventral vein was $0.90 \mathrm{~cm}$ and the most caudal and dorsal vein had a length of $2.40 \mathrm{~cm}$. The right kidney was $4.50 \mathrm{~cm}$ long, $2.63 \mathrm{~cm}$ wide and $2.58 \mathrm{~cm}$ thick.

No vascular variations regarding the left kidney were found.

\section{DISCUSSION}

The most accepted theory regarding the circumcaval ureter is that this variant originates due to an embryonic anomaly of the caudal vena cava. Several venous channels fuse and create several anastomoses that eventually give rise to the caudal vena cava. Some of these vessels can be seen near the metanephros and the developing ureters during ontogenesis $[2,5,6]$.

The right caudal cardinal veins should fuse and disappear, although, when this channel gives origin to the infrarenal portion of the caudal vena cava 


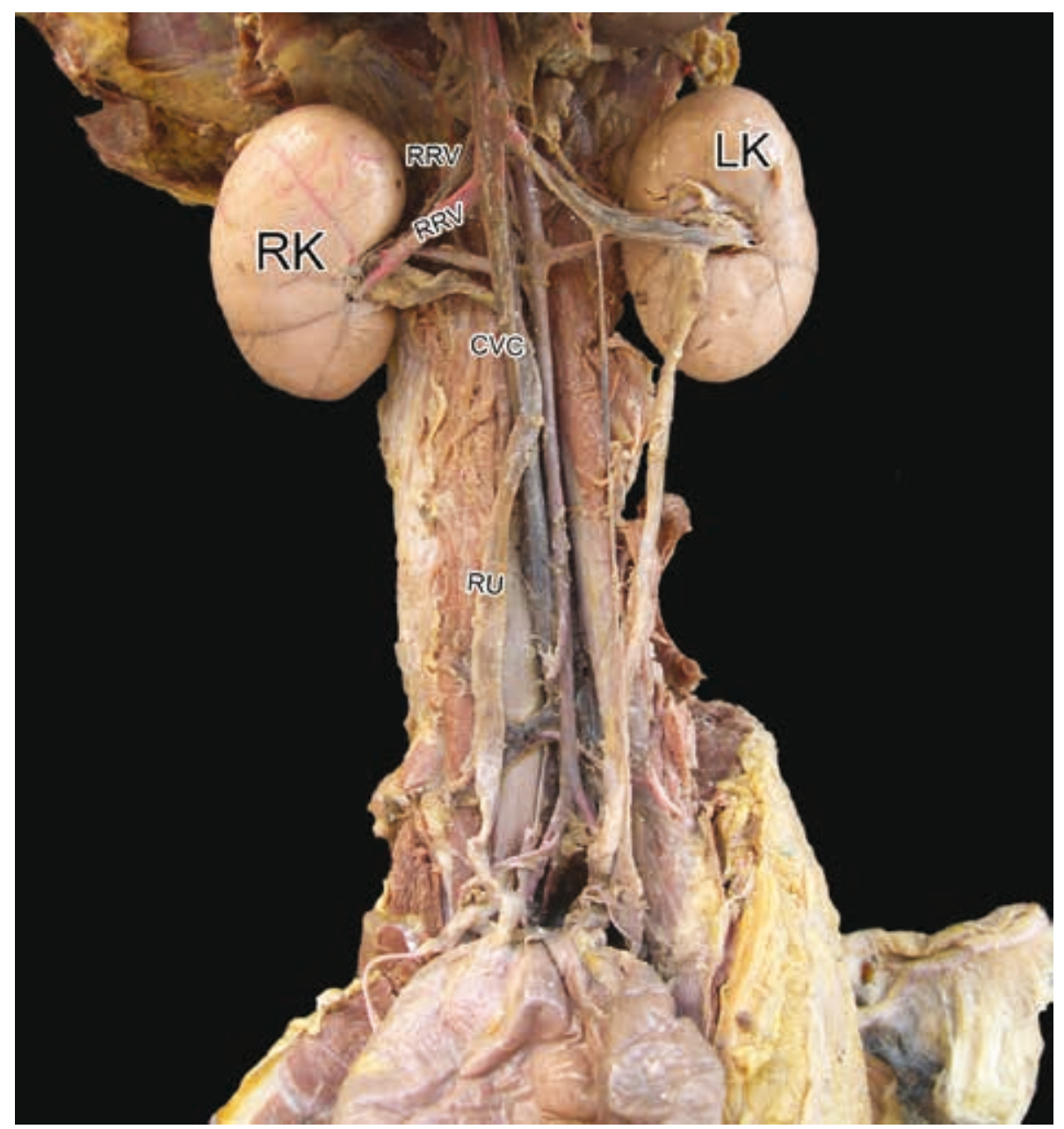

Figure 2. Ventral view of the second cat. The right circumcaval ureter (RU) can be seen together with the right renal veins (RRV); RK - right kidney; LK — left kidney; CVC — caudal vena cava.

instead of the right supracardinal vein, it causes the ureter to form a loop on the caudal vena cava $[5,6]$.

In cats, the circumcaval ureter is known to be a rare variant with few case reports in the literature $[3,5]$, although a study conducted by Bélanger et al. [2] observed the presence of a circumcaval ureter in $35.2 \%$ of 301 cats, while the majority of these variants were right-sided (30.6\%), while only $1.3 \%$ of the cats had a left circumcaval ureter.

In humans, this anatomical variation can be classified into two distinct types: type 1, in which the circumcaval ureter crosses posterior to the inferior vena cava near the third lumbar vertebrae, thus possessing an "S-shaped" aspect; and type 2, in which the aberrant ureter lies horizontally and immediately crosses posterior to the inferior vena cava, thus having a "sickle shaped" aspect. It is known that the type 1 is the most common between them [1, 2]. Both cats reported herein presented the type 1 circumcaval ureter.
As previously stated, this variant in humans is seen more often and may be associated with hydronephrosis, haematuria and recurrent urinary tract infections, although there has been no reports indicating this association in cats, although some authors observed ureteral strictures caused by this variant $[7,10]$. Nonetheless, it has been related to these symptoms in dogs $[3,7]$.

The association of a right circumcaval ureter with a doubled renal vein is scarcely reported in the literature. However, the variant ureter can be associated with other venous anomalies, such as the double caudal vena cava $[2,3]$.

Duplication of the renal veins is an often variation. A study conducted by Rieck and Reis [8] observed that $26.93 \%$ of 1000 cats had this variation, while only $1.25 \%$ had three renal veins. This duplicity is found more commonly on the right side due to embryological causes $[4,8]$. 
The presence of a circumcaval ureter may influence diagnostic procedures such as cystoscopy and ureteral surgeries (e. g. ureteral bypass, ureteral stent, uretero-ureteral anastomosis, and others types of anastomosis) [7], while clinically, it may cause lumen obstruction $[7,10]$. In addition, the presence of supernumerary renal veins can influence procedures such as transplants [4].

\section{CONCLUSIONS}

In conclusion, the retrocaval ureter is a rare variation with almost no recognition in domestic cats. Despite the fact that it is not associated with symptoms in this mammal, the presence of a retrocaval ureter may lead to iatrogenic errors during surgery and confusion during exams such as cystoscopy; therefore, vascular variations and ureteral variations should be identified pre-operatively.

\section{REFERENCES}

1. Bateson EM, Atkinson D. Circumcaval ureter: a new classification. Clin Radiol. 1969; 20(2): 173-177, indexed in Pubmed: 5771632.

2. Bélanger R, Shmon CL, Gilbert PJ, et al. Prevalence of circumcaval ureters and double caudal vena cava in cats. Am
J Vet Res. 2014; 75(1): 91-95, doi: 10.2460/ajvr.75.1.91, indexed in Pubmed: 24370251.

3. Casteleyn C, Cornillie P, Van Cruchten S, et al. Left retrocaval ureter around the ipsilateral limb of a double caudal vena cava in a cat. J Comp Pathol. 2015; 152(4): 313-316, doi: 10.1016/j. jcpa.2015.01.010, indexed in Pubmed: 25798957.

4. Cáceres AV, Zwingenberger AL, Aronson LR, et al. Characterization of normal feline renal vascular anatomy with dual-phase CT angiography. Vet Radiol Ultrasound. 2008; 49(4): 350-356, indexed in Pubmed: 18720765.

5. Cornillie $P$, Baten $T$, Simoens P. Retrocaval ureter in a cat. Vet Rec. 2006; 159(1): 24-25, indexed in Pubmed: 16816160.

6. Lesma A, Bocciardi A, Rigatti P. Circumcaval Ureter: Embryology. Eur Urol Suppl. 2006; 5(5): 444-448, doi: 10.1016/j. eursup.2006.02.008.

7. Pey P, Marcon O, Drigo M, et al. Multidetector-row computed tomographic characteristics of presumed preureteral vena cava in cats. Vet Radiol Ultrasound. 2015; 56(4): 359-366, doi: 10.1111/vru.12251, indexed in Pubmed: 25786990.

8. Rieck AF, Reis RH. Variations in the pattern of renal vessels and their relation to the type of posterior vena cava in the cat Felis domestica. Am J Anat. 1953; 93(3): 457-474, doi: 10.1002/ aja.1000930307, indexed in Pubmed: 13104340.

9. Testut $L$, Latarjet A. Tratado de Anatomía Humana. 8th ed. Barcelona: Salvat. 1958.

10. Zaid MS, Berent AC, Weisse $C$, et al. Feline ureteral strictures: 10 cases (2007-2009). J Vet Intern Med. 2011; 25(2): 222-229, doi: 10.1111/j.1939-1676.2011.0679.x, indexed in Pubmed: 21314722. 